\title{
PathfinderTURB: an automatic boundary layer algorithm. Development, validation and application to study the impact on in situ measurements at the Jungfraujoch
}

Yann Poltera et al.

Correspondence to: Giovanni Martucci (giovanni.martucci@meteoswiss.ch)

The copyright of individual parts of the supplement might differ from the CC BY 3.0 License. 


\section{Supplement}

\section{S1. PathfinderTURB algorithm and data}

The $S$ signal in eq.(1) in the accompanying article, is used by PathfinderTURB to retrieve the Convective Boundary Layer (CBL) and the Top of the Continuous Aerosol Layer (TCAL) at Payerne (PAY) and at the Kleine Scheidegg (KSE) with a time resolution of 1 minute (2 minutes at KSE) and a range resolution of $30 \mathrm{~m}$ (45 m at KSE). The $S$-profile is smoothed in space and time (2-D smoothing) using a Gaussian filter with FWMH equal to 5 bins in range corresponding to $150 \mathrm{~m}$ (225 $\mathrm{m}$ at KSE) and 5 steps in time corresponding to 5 minutes (10 minutes at KSE) and a standard deviation equal to 1.1 bins in range and 1.1 steps in time. Then smoothed with the 2-D Perona-Malik anisotropic diffusion method (Perona and Malik, 1990, as suggested in Haeffelin et al., 2012), based on 15 iterations and a conduction controlling coefficient of 2500. Values less than 1000 are set to 1000 and then the $\log 10$ is taken. The corresponding gradient field is calculated with central differences of this $\log 10$, smoothed $S$.

The central differences of the logarithm of $S$ in time and space are proportional to the vertical ratio of the logarithm of $S$ at two range bins for the time $t_{j}$ :

$$
\nabla\left(\log 10\left(S\left(R_{i}, t_{j}\right)\right)\right)=\frac{\left(\log 10\left(S\left(R_{i+1}, t_{j}\right)\right)-\log 10\left(S\left(R_{i-1}, t_{j}\right)\right)\right)}{R_{i+1}-R_{i-1}}=\frac{1}{R_{i+1}-R_{i-1}} \log 10\left(\frac{S\left(R_{i+1}\right)}{S\left(R_{i-1}\right)}\right)
$$

The criteria described in section 4.2 to restrict $S$ and $\nabla S$ in range and time are applied to PathfinderTURB at each time step and represent an important constraint for the algorithm. Next, we provide further details about the restrictions based on strong negative and positive gradients, as well as about the calculation of the atmospheric variance $(\operatorname{VAR}(S))$ and the TCAL. The last section describes the guidelines and data used for the human expert retrieval of the CBLH during the validation phase of PathfinderTURB in Payerne.

\section{S1.1 Strong negative gradients}

During the early morning period, the threshold is set to $\log 10(0.85) /(2 \mathrm{dR})$, which is the value of the derivative when $S$ undergoes a drop larger than $15 \%$. Else, the threshold is set to $\log 10(0.75) /(2 \mathrm{dR})$, (signal drop more than 25\%). The signal is analysed from $250 \mathrm{~m}$ upwards. In case there is a cloud obstruction the $\mathrm{CBLH}$, the limit is set to the cloud base height (CBH).

\section{S1.2 Strong positive gradients}

During the early morning period, the threshold is set to $\log 10(0.95) /(2 \mathrm{dR})$, which is the value of the derivative when $S$ undergoes an increase larger than $5 \%$. Else, the threshold is set to $\log 10(0.85) /(2 \mathrm{dR})$, (signal increase of more than 15\%). The signal is analysed from $250 \mathrm{~m}$ upwards. In case there is a cloud obstructing the ceilometer's view to the $\mathrm{CBLH}$, the limit is set at the $\mathrm{CBH}$. If a strong negative gradient occurs at less than $300 \mathrm{~m}$ above a strong positive gradient, the limit is set at the strong negative gradient's altitude. 
1 The strong negative gradients, strong positive gradients and lower altitude restrictions are applied to a time

2 resolution of 5 minutes, i.e. at time $t_{j}$, the restriction corresponding to the highest altitude over 5-minutes centred

3 on $t_{j}$ is retained.

4 S2. Calculation of the first transition to turbulence, atmospheric variance and total variance

5 The backscatter signal shows usually high temporal fluctuations at the top of the CBLH. However, when

6 calculating $\operatorname{VAR}(S)$ from the timeseries of $S$, mesoscale to microscale fluctuations contribute to the $V A R(S)$ value. We can use spectral analysis (FFT analysis) to try to recover the fluctuations solely due to aerosol number density fluctuations, but also to estimate whether we are in a fully developed turbulent regime, by looking at the spectral power decay. This gives, at each range and time step, an estimation of the atmospheric variability as an expression of turbulence.

Let us be given a $1 \mathrm{~h}$-long $S$ timeseries, centered at time step $t_{j}$, and at range $R_{i}$. The method, based on (Pal et al., 2013), goes as follows:

- De-spike the timeseries recursively with a sliding histogram as in (Lenschow et al., 2000). This step is used to remove extreme values due to, for example, clouds.

- De-trend with a polynomial regression of order 2 (i.e. subtract the fit from the timeseries).

- Calculate the FFT.

- Reduce the influence of mesoscale variability (periods of $30 \mathrm{~min}$ and more) with a high-pass filter applied on the FFT coefficients. We use a raised cosine filter (high-pass cutoff $f_{\text {cutoff }}=1 / 1800 \mathrm{~Hz}$ and roll-off factor $0.5)$.

- Integrate the power of the high-pass filtered FFT between $-0.75 f_{\text {Nyquist }}$ and $0.75 f_{\text {Nyquist. This gives us the }}$ atmospheric variance $\operatorname{VAR}^{a t m}(S)$.

- Integrate the power of the high-pass filtered FFT everywhere. This gives us the total variance $\operatorname{VAR}^{\text {tot }}(S)$.

- Perform a linear fit of the power spectrum, in logarithmic scale, between the 10-minutes frequency and the frequency corresponding to $0.75 f_{\mathrm{Nyquis}}$, and calculate the slope of the fit. Calculate then the relative error $e r r_{\text {rel }}$ of the slope compared to the theoretical value of $-5 / 3$ (Kolmogorov's power law for the inertial subrange) and apply the power 4 to this relative error.

$\operatorname{VAR}^{a t m}(S)$ is an hourly approximation of the atmospheric variance. We divide it by $\operatorname{VAR}^{\text {tot }}(S)$ and apply the power 4 to have a larger span of the values between 0 and 1, because this ratio of $\operatorname{VAR}^{a t m}(S)$ vs. $\operatorname{VAR}^{\text {tot }}(S)$ is about 0.75 for a signal with fluctuations dominated by noise and is close to 1 for a signal with fluctuations dominated by aerosol number density fluctuations. The resulting value, $\operatorname{VAR}(S)=\left(\operatorname{VAR}^{\text {atm }}(S) / \operatorname{VAR}^{\text {tot }}(S)\right)^{4}$, is a measure of the atmospheric variability.

In mixing and entrainment zones and periods, the $-5 / 3$ scaling law of turbulence for the inertial subrange can be observed. The relative error is then a good proxy to see if the region observed is in a turbulent regime or not. Again, we apply the power 4 to have a larger span of the values between 0 and 1 and so to better discriminate between low and high turbulence zones. The resulting value, turbfit $=\left(1-e r r_{r e l}\right)^{4}$, is a measure of turbulence. 


\section{S2.1 Implementation}

2 From 00:00 to 24:00, in steps of 10 minutes (in order to reduce the computational time), the atmospheric

3 variability $\operatorname{VAR}(S)$ and the turbulence turbfit are calculated over periods of one hour centered on each time

4 steps, for each altitude between the ground and the maximum climatological value set before.

The signal used is the standard signal $S$ (beta_raw) generated automatically by the CHM15K ceilometer, whose time and range resolutions are reduced to 1 minute and $30 \mathrm{~m}$, respectively. The Nyquist frequency $f_{\text {Nyquist }}$ is then 2 minutes and the first noise frequency $0.75 f_{\text {Nyquist }}$ is 2 minutes and 40 seconds.

The atmospheric variance $\operatorname{VAR}(S)$ and the turbulence turbfit are first smoothed in range with a running mean of 11 points (i.e. over $300 \mathrm{~m}$ ), then both in range and time (2-D) using a Gaussian filter with FWMH equal to 5 bins in range corresponding to $150 \mathrm{~m}$ and 5 steps in time corresponding to 50 minutes (11 bins in range and 11 steps in time for turbfit) and a standard deviation equal to 1.1 bins in range and 1.1 steps in time. Finally, they are interpolated on the time-and-range grid of the gradient field calculated before (i.e., $1 \mathrm{~min}$ and $30 \mathrm{~m}$ in PAY; 2 min and $45 \mathrm{~m}$ in KSE).

\section{$14 \quad$ S2.2 Inferior limit for altitude from transition to turbulence}

After the early morning period and before sunset, the altitude of the first transition corresponding to the onset of turbulence is calculated by applying a Haar-wavelet covariance transform to the profiles of turbfit and averaging it over multiple scales ( $60 \mathrm{~m}$ to $240 \mathrm{~m}$ at PAY, $90 \mathrm{~m}$ to $360 \mathrm{~m}$ at KSE), then we look at the first local minimum of the averaged transform.

If necessary, the retrieval of the CBLH could be made in an even more robust manner by varying the starting times and window widths, as described in the next subsection. However, doing so did not change much the results presented in the related article.

\section{S3 Robust choice of CBLH}

The pathfinder has an inherent uncertainty, and its sources are due to the a-priori range restrictions, the gradient and variance calculations, the window's width and the starting time. We consider here only the latter two uncertainty sources, and run the algorithm from sunrise to sunset with offsets of -30 to 30 minutes in steps of 10 minutes on the sunrise time, and window's widths of 15 minutes to 45 minutes in steps of 5 minutes. This 49 runs of PathfinderTURB, which increases the computational time to about $300 \mathrm{~s}$. Parallelization of this step is straightforward such that, if needed, the computational time could be reduced easily on nowadays standard multicore machines.

To select the final CBLH time series, we create a count matrix, which counts the number of CBLH candidates at a given altitude and at a given time step and divides this number by the number of CBLH candidates at this time step. This gives a value between 0 and 1. It is sometimes not enough to select the final CBLH time series based on the count matrix, and so we multiply the count matrix by a ratio matrix, which at each altitude and time step calculates the ratio of the mean of $S$ over $z+150 \mathrm{~m}$ to the mean of $S$ over $z-150 \mathrm{~m}$ for the considered altitude $z$. Negative ratios or ratios over 1 are set to 1 . We subtract then the ratio from 1 and multiply the result by 2 . This gives a value between 0 and 2 , values close to 2 belonging to clouds, else the values are mostly below 1 . 
1 For each CBLH time series, we add the values of the product of the count and ratio matrices along the time

2 series path. The time series having the maximal value is then selected as final CBLH time series.

\section{S4. Calculation of the TCAL}

The same time resolution and the 2-D Gaussian smoothing applied to the $S$ signal (Sect. S1) are applied also to the detected noise level by using error propagation and ignoring non-diagonal covariance terms. The signal-tonoise ratio (SNR) is then calculated simply dividing $S$ by the noise level.

The signal is considered too noisy when the SNR drops below 0.6745 or when $S$ becomes artificially negative (which happens due to the manufacturer automatic background light subtraction procedure). This creates a binary mask the same size of $S$ with the limitation that isolated points are not seen as noisy even if they have neighbouring noisy points and vice versa. Following (Biavati, 2014), image processing morphological operators, three erosions followed by 20 dilations, are used to make such isolated points disappear. Then, at each time step, the SNR mask is set to 0 at and above the first range bin above $600 \mathrm{~m}$ where the mask is 0 .

The discrimination of pure molecular signal from the aerosol signal is performed here by setting a threshold value on the profile of $\log 10(S)$. Once we have determined the instrumental calibration constant using the procedure suggested by (Wiegner and Geiß,2012) and after we have calculated the molecular profiles over one year with the formulas given in (Bucholtz, 1995) (Pressure and temperature from radiosounding), a threshold $T h$ to discriminate aerosols from molecules can be derived as $T h=4.625$. We then define a binary mask for aerosols based on the threshold $T h$. Each profile of $\log 10(S)$ is smoothed in range by applying a 11-point running mean at all ranges above the first range bin where the overlap function is larger than 0.05 , and the aerosol mask is set to 0 at and above the first range bin where the running mean is lower than $T h$. The aerosol mask is also set to 0 at all ranges above the detected cloud base. Then, three erosions followed by 10 dilations are applied to the mask. For profiles where $S$ is already smaller than $T h$ at the ground, the mask is set to 0 at all ranges (purely molecular atmosphere). At each time step, the TCAL is given by the first range bin where the product of the SNR mask and of the aerosol mask is 0 . The actual temporal resolution of the calculated TCAL is then decreased to 10 minutes (5 time steps), i.e. for each time step, the highest TCAL calculated during 5 time steps centred on the current time step is selected. In case of low cloud, the TCAL is not considered for the analysis.

\section{S5. Human expert CBLH retrieval in Payerne}

The following guidelines were adopted to perform the retrieval of the CBLH by human experts:

- A CBLH detection must lie on or be very close (within \pm 2 range bins) to an aerosol gradient.

- A CBLH detection must always exist during clear-sky periods. It is however subject to restrictions in presence of cloud, fog and precipitation:

- Cloud: the detection must match the cloud base.

○ Fog or low stratus cloud (<200 m a.g.1.): detection allowed when it starts to dissipate.

- Precipitation: detection possible only during time intervals without precipitation of at least 2 hours.

- Meteorological and astronomical information should be taken into account in order to respect the following criteria: 
- The detection starts at sunrise and ends at sunset.

- The CBL starts developing from the ground after sunrise, i.e. elevated layers shall not be selected during early morning stage.

- The CBLH shows a decay/breakdown when the vertical heat flux at the ground drops to about 0 and $\Delta T$ has a negative local minimum value normally at mid-afternoon till the end of the day, with $\Delta T$ calculated hourly by taking the difference between two consecutive hourly means of the surface temperature measurement. If no decaying layer can be followed, no detection is to be provided.

- The expert selects as many points as it is necessary in order for a linear interpolation between two adjacent points to represent trustworthily the short-term evolution of the observed layer.

All information are taken into account (station measurements, CBLH estimations from remote-sensing instruments and radio-sounding launches, synoptic conditions, etc.) and compared to the expert detection. In case of clear mistakes by the expert, the detection points should be edited accordingly.

\section{S5.1 Determination of the expert consensus}

The CBLH detections by each expert are linearly interpolated over $30 \mathrm{~s}$ and truncated before sunrise and after sunset. Before comparing the CBLH values retrieved by PathfinderTURB with those detected by the experts, a number of additional restrictions were defined. Manually-detected CBLH values for a given day must fulfil the following conditions in order to be "valid":

- No precipitation (station measurement) and no fog (ceilometer measurement) for more than 2 consecutive hours.

- $\quad$ Only time periods of at least 30-minute duration, containing interpolated data from at least two experts and with spread (i.e. difference between the maximum and minimum values) of less than $10 \%$ of the mean CBLH plus $100 \mathrm{~m}$ are taken into account. The allowed spread increases with altitude because the probability to lie on different layers decreases with increasing altitude. The offset of $100 \mathrm{~m}$ added to the maximum allowed spread is an empirical value that translates into a permitted $300-\mathrm{m}$ spread for a CBLH at $2000 \mathrm{~m}$, which is a conservative estimate of the depth of the entrainment zone at that altitude (EZ depth can be $40 \%$ of the CBLH).

The CBLH detections respecting the above criteria are retained for comparison with PathfinderTURB. The mean value of all the valid detections (reference and test group) for each time step was calculated along with the lower and upper error bounds determined as the minimum and the maximum detected CBLH. In total, an expert consensus was reached for 135 days, out of the initial 174, covering a total of 43914 minutes. On 6 days, no ceilometer data were available. On 13 days, poor weather conditions prevented all detections. On 20 days, either the spread was too large or the duration of the matched temporal interval was too short.

Because the number of CBLH detections decreases as the days become shorter in winter, midday is the period of the day with the highest availability of detections (and the best agreement). Both morning and afternoon periods present difficulties, when detecting the CBLH from the timeseries of S, nevertheless the morning provides better availability of detection than the afternoon. The limitations of morning detections are related to the fact that during the first 2-3 hours after sunrise the CBL top is still low above the ground $(<200 \mathrm{~m}$ a.g.1.) and the overlap- 
1 corrected, normalized signal, $\mathrm{S}$, is affected by the incomplete overlap in that zone, which makes the detection in 2 the first few hundred meters difficult and more uncertain. Another source of complexity in the morning 3 detections is that the residual nocturnal layer may remain aloft and very close to the developing CBLH, which 4 increases the uncertainty related to the layer attribution. During the late afternoon, the CBL transforms into the $5 \quad \mathrm{RL}$ as soon as buoyancy weakens and it becomes neutrally stratified. Under these conditions, the CBL top drops 6 rapidly, but usually without displaying a clear aerosol gradient, which leads to a large (and somewhat 7 unphysical) spread among the experts' detections. 